\title{
IUE AND MASSIVE BINARIES
}

\author{
D. J. Stickland \\ Space Science Department \\ Rutherford Appleton Laboratory \\ Chilton, Didcot \\ Oxfordshire, OX11 0QX, UK
}

\begin{abstract}
The role of IUE in the determination of orbital elements of massive stars is reviewed, emphasising the complementary value of ultraviolet observations when combined with archival optical data. Results on eight systems are described and the potential of the IUE archive for radial velocity work in general is advocated.
\end{abstract}

\section{Introduction}

The factor dominating, above all others, the evolution of a star is its mass; and the only certain way of deriving stellar masses is through the determination and interpretation of binary star orbits. In the case of the populous low-mass stars, statistical analysis of large quantities of such data is feasible, but for the rarer massive stars, it becomes important to study closely the individual cases, in combination with other astrophysical data, in order to estimate as accurately as possible the masses.

Spectroscopic binary work has long been the province of optical astronomers, as inspection of the Eighth Catalogue (Batten et al. (1989)) will attest. However, the archive of high-resolution spectra secured by the International Ultraviolet Explorer (IUE) now offers an additional, and in many ways complementary, source of data to be exploited. As this archive continues to build up, so it becomes increasingly worthwhile to investigate ways of using it to firm up our data base on the masses of the biggest stars.

\section{Why use IUE?}

Astronomy is very prone to fashion and I think it is fair to say that the determination of spectroscopic binary star orbits is not one of the trendy pursuits at the present time. So why waste a uniquely valuable facility like IUE getting spectra for something that can surely be done from the ground? Part of the answer, of course, is that virtually all of the binary orbit work done so far has been undertaken using archival data which were secured initially for some far more worthy(?) cause.

However, there are valid reasons for taking ultraviolet spectra for radial velocity purposes. One such is when one needs to avoid spectral contamination by a companion which is causing problems in the optical (eg, dragging of the spectrum at phases near the systemic velocity), but which might not, by virtue of a lower temperature, show up in the ultraviolet. Fig. 1 shows optical data on $\iota$ Ori where dragging is evident; it seems to be 
Fig 1: The optical (RGO) and uv radial velocity data and orbital solutions for Ł Ori.

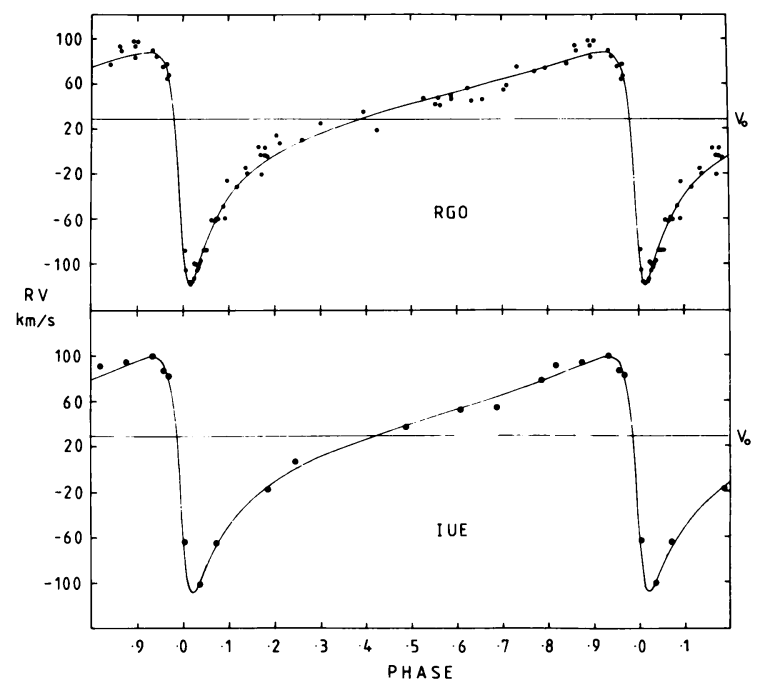

less marked in the uv data. A more extreme case is when one component cannot be seen at all in the optical and one must hunt for it in the uv.

Another justification is the richness of the photospheric spectrum in the far ultraviolet in comparison with the optical; furthermore, many of the lines that are found in the visible part of the spectrum, and especially those of hydrogen and helium, are prone to disturbance by wind effects. An example of the utility of IUE data here might be the case of V 861 Sco, where optical studies show different lines giving different velocity curves; cross correlation on the rich far-uv spectrum yields a very satisfactory result.

Finally, although perhaps not a sound reason for obtaining new IUE observations, is the modern epoch of this class of data. Much of the pioneering, and indeed definitive work was done in the first half (if not first quarter!) of this century and there are considerable gains to be had in a re-examination and comparison of orbital parameters over a long time base. Perinds can be improved, possible changes of period may be detected, and apsidal motion may be measured. The latter two are of considerable significance for massive stars, the first being related to mass loss or exchange and the second to the internal structure of these rapidly evolving stars.

Thus we see that IUE can play a useful role in binary studies in addition to the other tasks of determining the physical conditions that we all know it can do so well. Many investigations can be started right away using the vast archive of over 70,000 images already in the public domain. At least 10,000 are high dispersion spectra of massive stars (mid-B type or earlier) and in numerous individual cases, extensive data runs are available. Although a senior citizen by space experiment standards, it is to be hoped that a few more years of productive service can be anticipated and that the Final Archive of optimally reprocessed IUE data, now being planned by the three agencies involved (NASA, ESA, and SERC), will provide rich pickings for years to come. 
Fig 2: All available radial velocity data and the solution for HD 37017; the IUE data are represented by open symbols

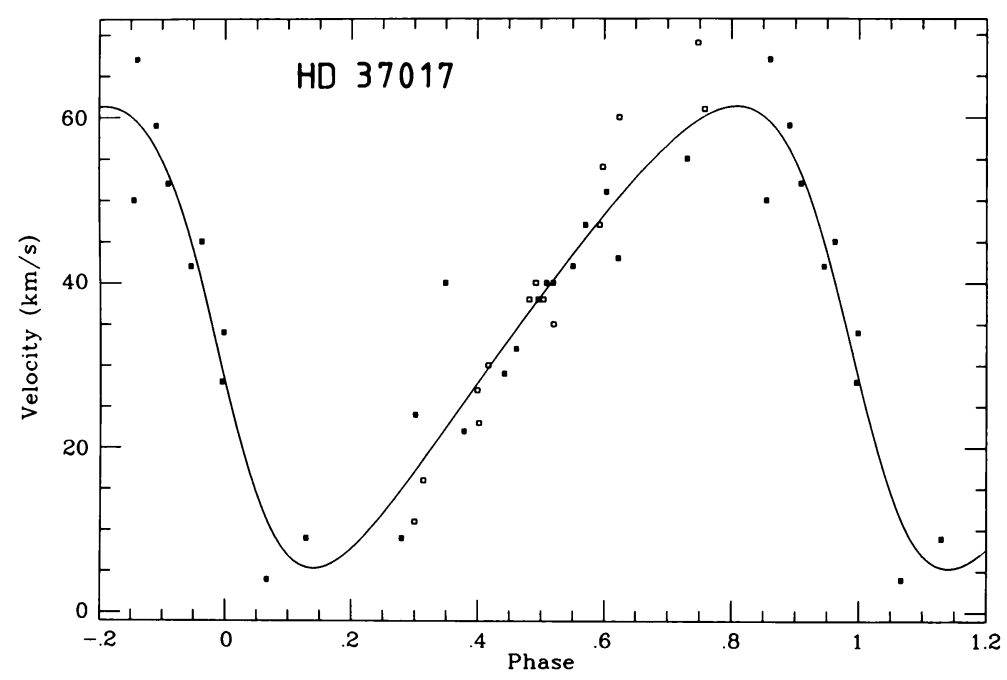

\section{Measurements: problems and solutions}

In common with most modern detection systems, but unlike the traditional long slit spectrographs, the high-resolution mode of IUE does not admit the impression of both stellar and wavelength calibration spectra on the same image and one does not, therefore, have an immediate reference system against which to measure wavelengths. The wavelength scale is imposed during image processing on the basis of a set of dispersion constants for the echelle spectrograph evaluated from measurements of the positions of lines in the Platinum spectrum secured from time to time as part of the calibration programme. This spectrum is produced by a $\mathrm{Pt}-\mathrm{Ne}$ hollow cathode lamp mounted behind the primary mirror and whose light is reflected into the spectrographs off the back of the Sun shutter; since it is inadvisable to operate both lamps and mechanisms more often than is absolutely necessary, it is impossible to take calibration spectra along with every programme spectrum. Thus spectrograph (plus image processing) instabilities (ie, zero point drift) cannot be monitored routinely. Furthermore, most spectra these days are secured using a large aperture $(10 \times 20$ arcsec $)$ and although the placement of the (3 arcsec diameter) image within it is generally found to be accurate, it cannot be guaranteed in this slitless system.

How then are we to proceed in order to get radial velocities that can be used in the determination of binary orbits? Probably the best approach for hot stars begins with the recognition of the relatively rich spectrum of interstellar lines to be found in the far ultraviolet and the assumption that their radial velocity is constant in a given star. It may turn out that in many stars such lines are blends of several components, but provided that the same set is used and measured consistently this is not a problem; in this regard, care has to be exercised when producing a set for spectra taken both with the large and small apertures. Laboratory wavelengths (in vacuum) can be applied to these lines (eg. from Morton \& Smith (1973)) or an empirical set can be established for use on a particular star. 
One merit of using the interstellar lines is that they will share with the stellar spectrum the displacements due to the motion of the Earth and IUE and thus normalization to the interstellar velocity (which can be an arbitary value or that determined by ground-based observers) eliminates the need to evaluate heliocentric corrections.

Proceeding now to the stellar spectra, one is confronted with the problem that ultraviolet astronomy is a fairly youthful science and that approved lists of lines for velocity measurement, such as have been thoughtfully provided in the optical region over many years, just do not exist. Certainly the identifications of some of the stronger features (particularly lines formed in winds) are safe enough, but the same cannot be held true of the bulk of "photospheric" lines. The lazy way to circumvent this hazard is to ignore identifications and to proceed to use one spectrum as a template for the others, taking a selection of $\mathbf{3 0}$ or so well defined, consistently-measurable absorption lines, and recording differential velocities with respect to that template. Initially I did this in a rather tedious way, setting on each line with a cursor and recording the wavelength for later computation of the velocity shift. Latterly, however, Ian Howarth has provided me with a nice cross-correlation routine which takes the drudgery out of it and gives essentially identical results; it skips all the wind and interstellar lines but gives much more spectrum than I would have measured manually.

All this is very well but it enables only relative velocities to be obtained. This is fine for many purposes, as will be shown later, but when comparing results from IUE with those from ground-based work, it can be of considerable interest to have a reliable value for the systemic velocity. To this end, work has begun, under the auspices of the UK IUE Project, to establish laboratory-based line lists for use with high-resolution spectra of hot stars. At present, a list has been prepared for SWP spectra of early B-type stars based on $\zeta$ Cas (B2IV); this will shortly be joined by one for O9I stars based on HD 209975; this turns out to be a rather sharp-lined star with constant velocity. It is expected that LWR/LWP lists will follow (although the time scale is not clear!). If, then, the uv interstellar line frame can be tied in to optical measures on a given star, absolute stellar velocities can be achieved, probably to within about $5 \mathrm{~km} / \mathrm{s}$.

Alternatively, one can pin one's hopes on the IUE calibration and image processing teams and assume that the extracted spectrum has a correct wavelength frame (that of the satellite before 1981 November, heliocentric thereafter) and derive absolute velocities directly, perhaps using the interstellar lines as a check. Work to check out this approach is still underway but it seems probable that a good system will be available in the Final Archive, and that it is actually not too bad at present. The importance of this is in the application it may have for cooler stars where the interstellar spectrum may not be easy (or even possible) to measure.

\section{Some case studies}

The purpose here is to highlight some of the advantages of using IUE spectra with examples studied thus far. The astrophysical discussion is not exhaustive and readers are referred to the original papers for more detail.

\section{$\iota$ Orionis}

The first in a series of papers to appear in The Observatory making use of IUE data for orbital studies was on $\iota$ Ori (Stickland (1987a)); many of the problems discussed above are treated therein. Fig. 1 shows the orbit derived from 19 SWP spectra, together with that produced from optical data at comparable resolution. Although the IUE data set was 
Fig 3: The apsidal motion of $\delta$ Ori; the most recent point is from IUE data (see Harvey et al. (1987) for a description of the various fits to the data).

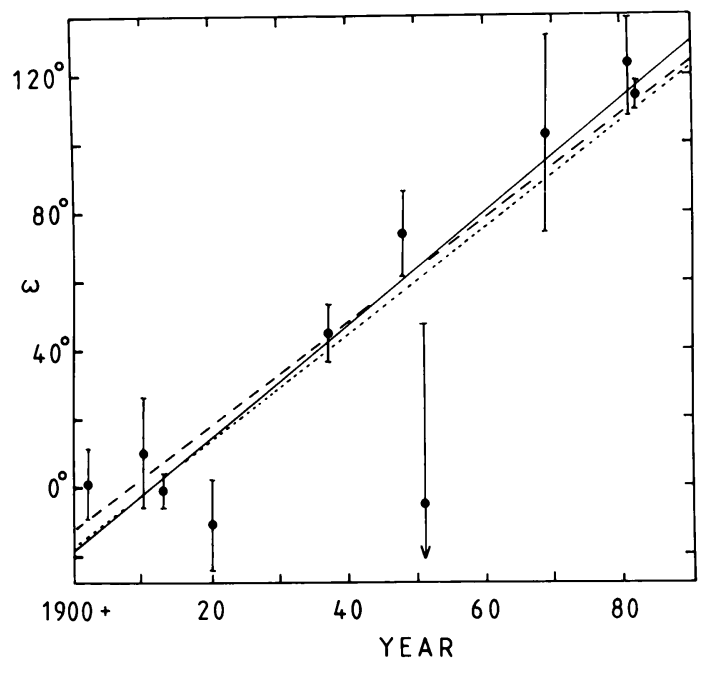

rather limited, particularly around periastron, there are suggestions that the noticeable dragging near the systemic velocity in the optical data is less marked in the SWP spectra. Given the spectral types of O9III and B1III for primary and secondary respectively (Stickland et al. (1987)), it is not surprising that the uv spectrum of the primary is less contaminated.

\section{Plaskett's Star}

It has been clear for some time that the more massive the star, the stronger the desire seems to be to lose mass. In binary systems, prolific mass loss can result in an increase of period and this can be monitored when a series of data sets are available over a long time scale. In the case of the star with the largest known mass (actually a lower limit on the secondary of about $\left.60 \mathrm{M}_{\odot}\right)$ - Plaskett's Star $(\mathrm{O} 8 \mathrm{e})$ - the period appears to be constant to better than about 0.00004 days on a time scale of decades (Stickland (1987b)); it has to be said, however, that the observed estimates of the mass-loss rate, $5 \times 10^{-6} \mathrm{M}_{\odot} \mathrm{yr}^{-1}$, are just inside this constraint.

\section{HD 37017}

Fourteen IUE spectra were used in a hitherto unpublished study of HD 37017 (B1.5V). Earlier work by Blaauw \& van Albada (1963), which included recognition of a few archival data from Victoria (Plaskett \& Pearce (1931)), gave only a rather approximate period of 18.65 days. Unfortunately the IUE data were all concentrated on the ascending branch of the velocity curve although they do spread far enough along it that the relative IUE velocities may be tied into the system of Blaauw \& van Albada fairly well. This enables the period to be refined somewhat and the orbital elements derived from all the available measures are presented below, with the graphical representation given in Fig. 2. A more accurate appraisal will be possible if absolute IUE velocities are derived, as should be 
Fig 4: The radial velocity curves of $\gamma^{2}$ Velorum; filled symbols represent the WC8 star and open ones the O9I component

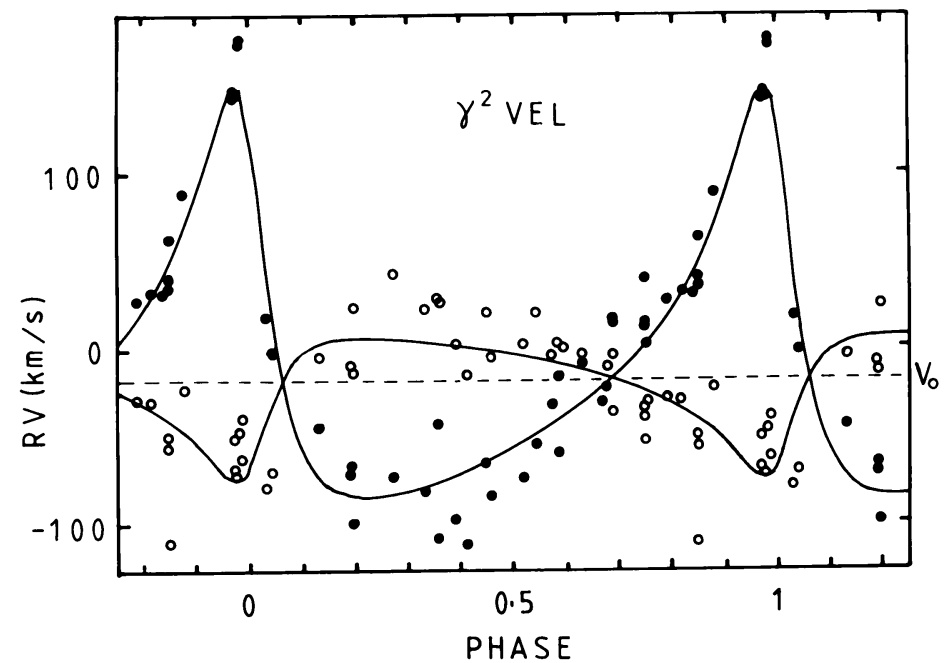

possible employing the $\zeta$ Cas line list.

Orbital elements for HD 37017

$P=18.65245 \pm 0.00043$ days

$\mathrm{T}_{o}=\mathrm{JD} 2435002.91 \pm 0.60$

$\mathrm{K}=28.9 \pm 2.0 \mathrm{~km} \mathrm{~s}^{-1}$

$\mathrm{e}=0.27 \pm 0.05$

$\omega=100 \pm 13$ degrees

$\gamma=34.9 \pm 1.2 \mathrm{~km} \mathrm{~s}^{-1}$

r.m.s $=5.9 \mathrm{~km} \mathrm{~s}^{-1}$

\section{$\delta$ Orionis}

Among the most satisfying of the IUE binary studies to date (Harvey et al. (1987)), $\delta$ Ori (O9.5I) yielded results better (in terms of r.m.s. residual) than all of the many previous optical investigations. The value of this modern result was that it enabled a tight constraint to be put on the apsidal motion in this system, $1.67 \pm 0.11$ degrees per year, as shown in Fig. 3. Taking other system parameters from Koch \& Hrivnak (1981), masses of 23 and $9 \mathrm{M}_{\odot}$ can be deduced with radii of 17 and $10 \mathrm{R}_{\odot}$, from which apsidal constants can be deduced which agree remarkably well with theoretical predictions.

\section{AO Cassiopeiae}

AO Cas $(08.5 \mathrm{e}+$ ?) presents numerous problems to the would-be determiner of orbits, as reviewed by Stickland \& Lloyd (1988); the stability of the period and the eccentricity have both been questioned while the mass ratio is a vexed matter indeed. The IUE results add weight to the notion of a constant period and are consistent with $\mathrm{e}=0$; 
Fig 5: The radial velocities and solution for $V 861$ Sco

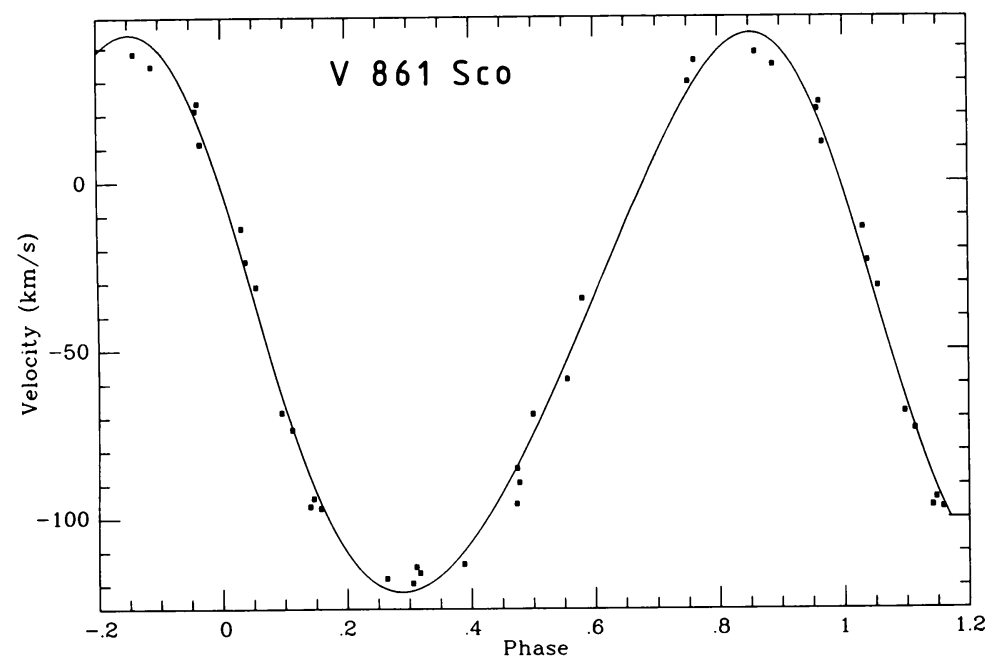

as to the mass ratio, this hinges on whether what has been deemed to be the secondary is really just that. Features were measured on the SWP spectra which appeared to move in antiphase with the primary lines, in the mainstream of optical results, and leading to $q$ $=1.32$. The implication is that we are perhaps seeing a system in the full swing of mass exchange, but it feels somehow uncomfortable!

\section{Canis Majoris}

$29 \mathrm{CMa}$ is the prototype Of star (now O8.5If) and was put on the IUE binary programme because of the high rate of mass loss from such objects and the consequent interest in period changes. 28 spectra were available for study and, in combination with results from the past - going back to 1916 - a tight constraint was put on this parameter: 4.393411 \pm 0.000003 days. The IUE paper (Stickland 1989), however, does contain an unfortunate error in the time of periastron given for the IUE data: it should have been 2443614.063. At the same time, the uv data perhaps yield the best value of $K_{1}$, giving a mass function of $5.5 \mathrm{M}_{\odot}$.

\section{$\gamma^{2}$ Velorum}

Perhaps the major challenge to date and the first double-lined system to be treated, the Wolf-Rayet $\gamma^{2}$ Vel presented a number of problems and gave the least tidy results (Stickland \& Lloyd (1990)). Several absorption lines in the spectrum of the WC8 star were recorded and a few in the O9I which seemed to move in antiphase (Fig. 4). An important point in favour of the IUE work was the measurement of the N III lines at $\lambda 1750$ which could not have arisen anywhere else than the O9I star; this appeared to confirm the lower velocity amplitude of the $\mathrm{O}$ star found by Pike et al. (1983) rather than that of Niemela \& Sahade (1980). That said, the true elements of orbit are still clouded in mystery and dispute! 


\section{861 Scorpii}

V 861 Sco (B0Ia) is the first to benefit from the cross-correlation method. An orbit was obtained, on the basis of 26 spectra, which compares well in most respects with that secured by Wolff \& Beichman (1979), although the value of $\omega$ is markedly different and more in line with that deduced by Hill et al. (1974) several years earlier. However, before wildly speculating about rapid apsidal advance, it has to be noted that the IUE and Wolff \& Beichman observations overlap in time. Thus observational error coupled with the small eccentricity is probably the culprit; the r.m.s. residual for the IUE result is again the best. The new elements are set out below and the data displayed in Fig. 5.

\section{Orbital elements for $V 861$ Sco}

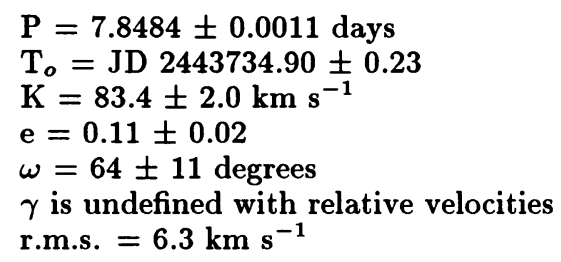

\section{Conclusions}

Although IUE has exceeded all expectations several times over, and many of us have come to take it for granted, the reality is that we will not have access to it for ever! So, if enthusiasts in the hot star community can see vital applications, in the area of massive binary stars, which have not already been treated, and suitable archival data do not yet exist, I exhort them to apply for time in the very next round. On the other hand, if your favourite star has been observed, why not see if you can extract radial velocities from the archival data - they may throw up some surprising results.

\section{References}

Batten, A. H., Fletcher, J. M. \& MacCarthy, D. G. (1989): P.D.A.O., 17

Blaauw, A. \& van Albada, T. S. (1963): Ap.J., 137, 791

Harvey, A.S., Stickland, D. J., Howarth, I. D. \& Zuiderwijk, E. J. (1987): The Observatory, 107, 205

Hill, G., Crawford, D. L. \& Barnes, J. V. (1974): P.A.S.P., 86, 477

Koch, R. H. \& Hrivnak, B. J. (1981): Ap.J., 248, 249

Morton, D. C. \& Smith, W. H. (1973): Ap.J.Suppl., 26, 333

Niemela, V. S. \& Sahade, J. (1980): Ap.J., 238, 244

Pike, C. D., Stickland, D. J. \& Willis, A. J. (1983): The Observatory, 103, 154

Plaskett, J. S. \& Pearce, J. A. (1931): P.D.A.O., 5, 1

Stickland, D. J. (1987a): The Observatory, 107, 5

Stickland, D. J. (1987b): The Observatory, 107, 68

Stickland, D. J. (1989): The Observatory, 109, 74

Stickland, D. J. \& Lloyd, C. (1988): The Observatory, 108, 174

Stickland, D. J. \& Lloyd, C. (1990): The Observatory, 110, 1

Stickland, D. J., Pike, C. D., Lloyd, C. \& Howarth, I. D. (1987): A. \& Ap., 184, 185

Wolff, S. C. \& Beichman, C. A. (1979): Ap.J., 230, 519 